\title{
Flavonoids-The Most Potent Poly-phenols as Antidiabetic Agents: An Overview
}

\author{
Saritha Marella* \\ Department of Nanotechnology, Acharya N.G. Ranga Agricultural University, India \\ *Corresponding author: Saritha Marella, National Post doc fellow, Nanotechnology Laboratory, Regional Agricultural Research Station, Acharya N.G \\ Ranga Agricultural University, Tirupati- 517502, A.P, India
}

Submission: 㠿 October 24, 2017; Published: 眥 December 06, 2017

\begin{abstract}
Diabetes mellitus is a metabolic disorder of carbohydrate, lipid and amino-acids characterized by polyuria, polyphagia, polydypsia, blurred vision etc. It is the most prevailing disease affecting nearly 285 million people worldwide and is expected to rise to 438 million by 2030 . In spite of numerous drugs currently available in the treatment of diabetes, herbal products tend to gain much importance over any other due to less/minimal adverse effects in recent decades. Around $60 \%$ of the world's population is using medicinal plants as a traditional medicine out of which India is major contributor. Polyphenols, in particular flavonoids can be suggested as better therapeutic agents in the management of diabetes mellitus and also chronic complications associated with the disorder. In the present review, focus is made on various flavonoids, their chemical nature and their mechanisms of action from various plant sources in overcoming hyperglycemia and its associated complications.
\end{abstract}

Keywords: Antidiabetic; Flavonoids; Glucose tolerance; Hyperglycemia; Poly-phenols

Abbreviations: AGEs: Advanced Glycation End Products; CAT: Catalase; DM: Diabetes Mellitus; GLUT4: Glucose Transporter4; GPx: Glutathione Peroxidise; HbA1c: Glycated Haemoglobin; PPARy: Peroxisome Proliferator-Activated Receptor Gamma; ROS: Reactive Oxygen Species; SOD: Super Oxide Dismutase; SREBP-1c: Sterol Regulatory Element-Binding Transcription factor 1

\section{Introduction}

Natural phytochemicals available have a wide range of therapeutic efficacy as they exhibit numerous biological activities in the treatment of chronic diseases. These phytochemicals attract the attention of public as they are free/minimal side effects on consumption for long term treatment of diseases compared to any other modern medication [1]. Flavonoids are an important class of plant based compounds found in many fruits, vegetables, legumes, grains, nuts and have a wide range of actions and medicinal uses too. They are hydroxylated phenolic substances and the hydroxyl group mediate their antioxidant effects by scavenging free radicals by chelating metal ions [2]. They form the biggest group of poly-phenols in plants with health beneficiary like preventing degenerative diseases like cancer, cardiovascular, metabolic and neurodegenerative diseases. As "Redox Regulators", they are even more powerful than the common antioxidants like Vitamins $\mathrm{E}$ and $\mathrm{C}$ in preventing free radical mediated cellular damage. They are found to interfere with the digestion, absorption, and metabolism of carbohydrates and also evident to have anti-inflammatory, antiallergic, antiviral and anticancer properties [3].

\section{Chemical nature of flavonoids}

The chemical nature of flavonoids is usually a conjugation between the aromatic rings, glycosidic moieties, methoxy groups and other substituents. They contain conjugated double bonds and groups (hydroxyl or other substituents) that can donate electrons. Flavonoids with biological activity are often. called bioflavonoids. They possess the ability to capture superoxide, hydroxyl and lipid radicals and so flavonoid analogues and their metal complexes play a significant role in agriculture, industrial and pharmaceutical chemistry. Flavonoids are sub classified into flavones, flavonols, flavanones, flavonols, isoflavones and anthocyanidins based on differences in generic structure of the $\mathrm{C}$ ring, functional groups on the rings and the position at which the $\mathrm{B}$ ring is attached to the $\mathrm{C}$ ring. Structural diversity of flavonoids and their analogues are responsible for heterogeneity in activities [4] (Figure 1).

\section{Classification of flavonoids}

Flavanols: They have a hydroxyl group in position 3 of the $\mathrm{C}$ ring, is glycosylated. They are the most common and largest subgroup of flavonoids in fruits and vegetables in the range from monomeric flavan-3-ols (e.g. catechin, epicatechin, gallocatechin) to polymeric procyanidins (condensed tannins). They have two chiral centers in the molecule, on positions 2 and 3, then four possible diastereoisomers. Epicatechin is the isomer with the cis configuration and catechin is the one with the trans configuration. Each of these configurations has two stereoisomers, namely, 
$(+)$-epicatechin and (-)-epicatechin, $(+)$-catechin and (-)-catechin. $(+)$-Catechin and (-)-epicatechin are the two isomers most often present in edible plants. Catechins are usually found in the seeds and peels of unripened fruits. Green and black tea, red wine, dark chocolate, apricot, apples, peach, red raspberry, and blackberry are rich in catechins.

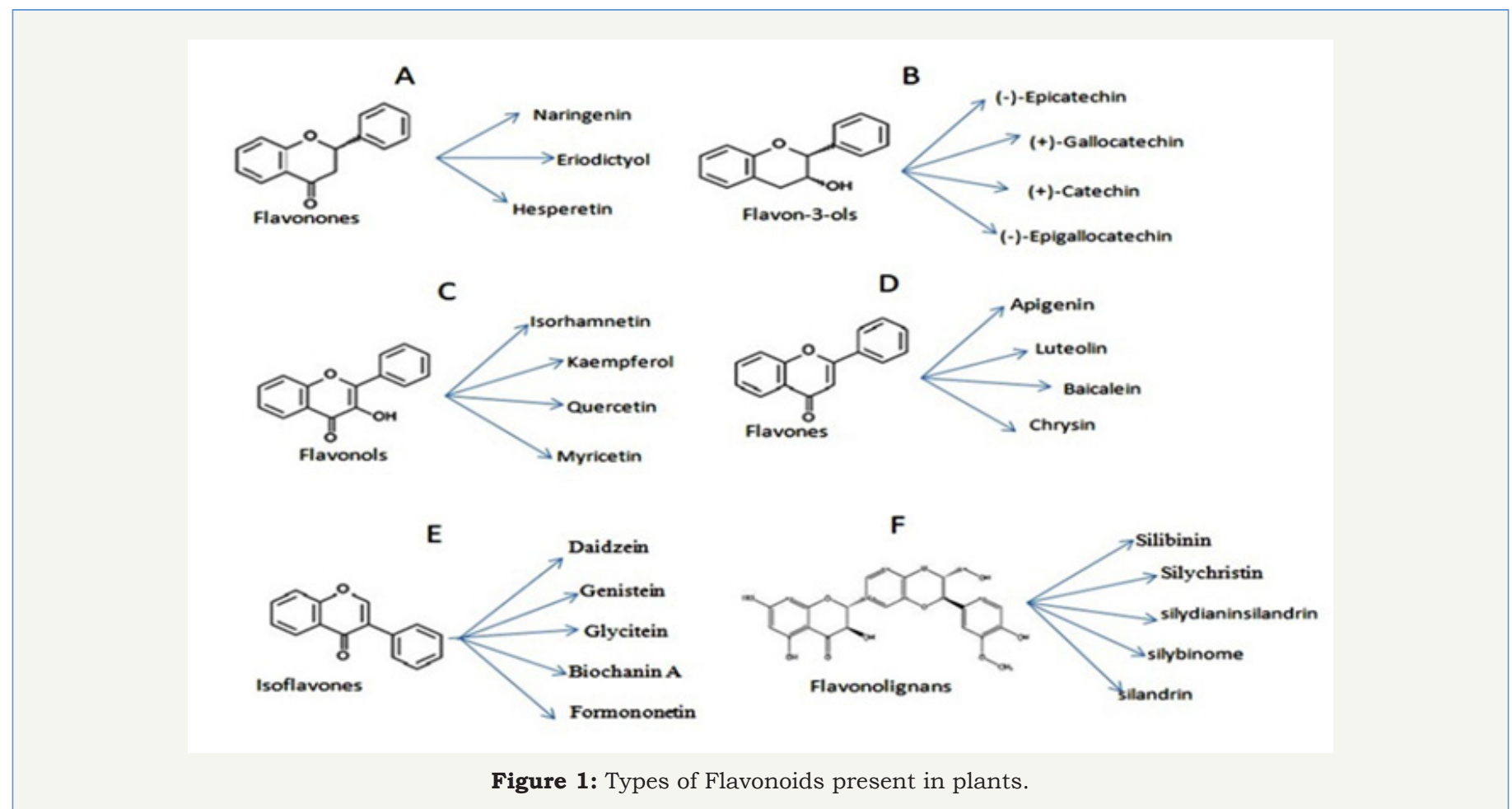

Anthocyanidins: Sugar units are bound mostly to position 3 of the $\mathrm{C}$ ring and they are often conjugated with phenolic acids such as ferulic acid. The color of the anthocyanins depends on the $\mathrm{pH}$ and also by methylation or acylation at the hydroxyl groups on the A and B rings. They exist as aglycone form which is structurally based on the flavylium or 2-phenylbenzopyrilium cation possessing hydroxyl and methoxyl groups present at different positions of the basic structure. These phytochemicals are natural pigments usually abundant in fruits and vegetables and responsible for their characteristic colours. Tea, honey, fruits, vegetables, nuts, olive oil, cocoa and cereals are rich in anthocyanidins.

Flavanones: Flavanones are also called dihydroflavones with the $\mathrm{C}$ ring saturated. The flavanones are multi-hydroxylated, and several hydroxyl groups can be glycosylated and/or methylated.

Some have unique patterns of substitution like furanoflavanones, prenylated flavanones, pyranoflavanones or benzylated flavanones and a great number of substituted derivatives. The efficacy of flavonols as antioxidant agents mostly depends on their chemical structure. The catechol structure in the B ring, is a radical target site; the 2,3-double bond in conjugation with a 4-keto function are responsible for electron delocalization from the $\mathrm{B}$ ring and the additional presence of both 3- and 5-hydroxyl groups have strongest radical absorption. They are found in almost all parts of plants i.e from vegetative to generative organs. Hesperidin is rich in Citrus, Naringenin is abundant in oranges, tomatoes etc.

Isoflavones: Flavonoids in which B ring is linked in position
3 of the ring $\mathrm{C}$ are called isoflavones. They constitute the 3-phenylchromen skeleton which is chemically derived from the 2-phenylchromen skeleton by an aryl-migration mechanism. They have structural similarities to estrogens, such as estradiol, and for this reason they are also called phytoestrogens. They are rich in legumes, in particular soy, besides peas, beans, clover sprouts, sunflower seeds etc. They have lipid lowering, antioxidant effects in blood vessels.

Flavones: They are structurally very similar to flavonol compounds, having an extra hydroxyl substitution at the carbon 3-position. The major flavones are included apigenin and luteolin. Luteolin usually is found in vegetables and fruits such as broccoli, celery, carrots, parsley, onion leaves, cabbages, peppers, chrysanthemum flowers, and apple skins and apigenin is found in onions, parsley, wheat sprouts, tea, oranges, chamomile, and in some seasonings.

Flavonolignans: The basic structure of flavonolignan consists of the dihydroflavanol taxifolin linked to coniferyl alcohol moiety through an oxeran ring. They are formed by oxidative coupling process between a flavonoid and a phenylpropanoid usually a conieryl alcohol. Coumarolignans found in some plants are formed by oxidative coupling mechanism of combination of coumarin with cinnamyl alcohol. The biological activity of silymarin lies in the oxeran ring and activity is lost on its opening. Silybin and isosilybin have the same trans conformation of C-2, C-3 and C-7', C-8'. 


\section{Mechanism of Anti hyperglycaemic activity of flavonoids}

Flavonoids of all sub-class have proven to have antidiabetic properties by (1) enhancing insulin secretion via regeneration of pancreatic $\beta$-cells, (2) enhancing insulin mediated glucose uptake by target cells, (3) inhibiting of aldose reductase (4) increasing $\mathrm{Ca}^{2+}$ uptake [5]. Flavonoids with potent antioxidant activity were shown to be effective in management of diabetes [6]. Antidiabetic activity of flavonoids depends on the chemical criterion (C-2-C-3 double bond and ketonic group at $\mathrm{C}-4$ position on ring $\mathrm{B}$ ) which is fundamental for the bioactivity of poly-phenol compounds [7]. These phytomedicines play significant role in maintaining blood glucose levels, glucose uptake, insulin secretory and immuno modulatory functions to prevent specific DM [8] (Figure 2).

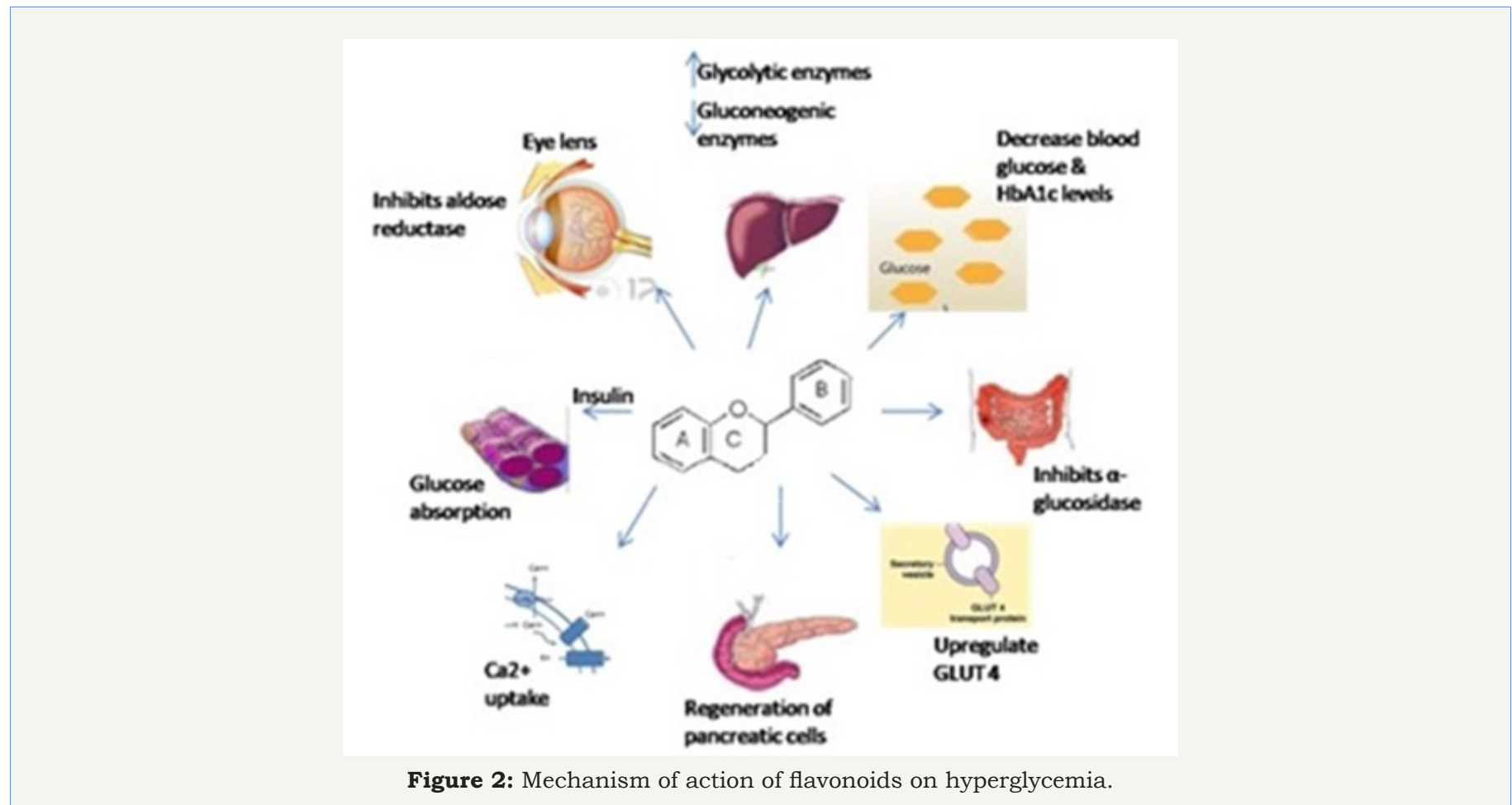

Flavanols: Catechins prevents oxidation and covalent modifications caused by free radicals in proteins [9]. Fisetin, a dietary flavonoid commonly found strawberry, apple, persimmon, grape, onion, cucumber and Cotinus coggygria Scop. improved the plasma insulin and antioxidant levels in diabetic rats and also significantly decreased the levels of blood glucose, HbA1c [10].

Anthocyanidins: They play a key role in cardiovascular disease, cholesterol decomposition as well as antioxidant efficacy and cytotoxicity. They protect DNA integrity and tissue antioxidant levels [11]. Anthocyanidins with one or more sugar moieties (anthocyanidin glycosides) are called anthocyanins. Proanthocyanidin oligomers formed from (+)-catechin and (-)-epicatechin subunits are called procyanidins cyanidin which is rich in berries improved insulin resistance, upregulated GLUT4 and decreased the hyperglycaemic condition in obese and diabetic mice [12]. Pelargonidin decreased the glucose and TBARS while enhancing the levels of SOD in STZ induced diabetic rats [13].

Diosmin (flavonoglycoside) usually found in citrus, exhibited anti-hyperglycemic activity in Streptozotocin-Nicotinamide induced diabetic rats after 45 days of treatment by enhancing the activity of glycolytic enzymes like hexokinase, glucose-6-phosphate dehydrogenase and also by inhibiting the gluconeogenic enzymes like glucose-6-phosphatase and fructose 1,6 bisphosphatase [14].
Rutin was demonstrated to have protective effect against hyperglycemia and dyslipidemia induced nephropathy, neuropathy, liver damage and cardiovascular disorders in addition to inhibition of sorbitol, ROS \& AGEs [15]. The underlying mechanism was supposed to be decreased carbohydrate absorption from small intestine, pancreatic $\beta$-cell stimulation to enhance insulin secretion, increased tissue glucose uptake and inhibition of tissue gluconeogenesis [16].

Flavanones: Hesperidin reversed the hyperglycemia and hyperlipidemia by down regulating free radical generation [17]. Naringenin significantly inhibited the intestinal $\alpha$-glucosidase activity thereby decreasing the post prandial blood glucose levels and also restored the lipid profile changes, improved antioxidant status and hepatic function markers [18].

Quercetin has antioxidant and anti inflammatory activities i.e protects blood vessels from damage by free radicals and also acts as anti histamine agent protecting from allergies. It also plays a major role in the sorbitol pathway by inhibiting key enzyme [19]. It reduced intestinal glucose absorption by inhibiting GLUT2, decreased lipid per-oxidation while improving the CAT, SOD and GPx levels during diabetes, also stimulating GLUT 4 expression in skeletal muscle and anti inflammatory effects on adipose tissue [20]. Specifically, the risk of diabetes was inversely correlated 
with the intake of flavan-3-ols (monomers and dimmers only) and flavonol [21]. Daily supplementation of flavan-3-ols and isoflavones (dar chocolate) to type 2 diabetics significantly improved insulin sensitivity and reduced the risk of coronary heart disesase in postmenopausal women [22].

Myrecetin significantly ameliorates insulin resistance besides antioxidant, anti inflammatory, aldose reductase inhibitory actions [23]. Administration of Kaempferol, an abundant flavonoid in berries, Gingko biloba, vegetables, grapes, to diabetic rats reverted the levels of blood glucose, insulin, enzymatic and non-enzymatic antioxidants [24].

Kaempferol also decreased the gene expression of PPAR- $\gamma$ and SREBP-1c in liver [25]. Hypoglycemic and hypolipidemic effects were investigated in high fat diet fed and Streptozotocin induced diabetic rats on treatment with flavonoids from tatary buckwheat (TBF) by Jing $\mathrm{Li}$ et al. [26]. Improved hyperglycemia, insulin resistance, glucose intolerance were observed besides normalizing the lipid and lipoprotein in the serum of diabetic rats on treatment for 30 days with Morin, a natural flavonoid commonly found in medicinal herbs like Prunus dulcis (Mill.) D.A. Webb, Chlorophora tinctoria (L.) Gaud., Psidium guajava L., fruits and wine [27]. Oral administration of Isorhamnetin, found in medicinal plants, such as Hippophae rhamnoides L., Oenanthe javanica (Blume) DC., and Ginkgo biloba L. Decreased sorbitol accumulation in lenses, R.B.C sciatic nerves and glucose levels in addition to regulation of expression of lipid metabolic enzymes [28].

Isoflavones: These are structural variants in which the shikimate derived aromatic ring is migrated to the adjacent carbon of the heterocycle. Flavonoids in the ethanolic extract of Sophora flavescens roots SF-EtOAc improved glucose tolerance and reduced hyperglycemia. The insulin levels were also resumed in diabetic mice after treatment further activating GLUT4 translocation supposed to be modulated by AMPK pathway [29]. Dietary intake of genestein significantly improved the lipid profile, plasma insulin, hyperglycemia in obese diabetic mice [30].

Flavones: Apigenin was efficient in overcoming hyperglycemia and reduced antioxidants like SOD, CAT, GSH in alloxan induced diabetic rats [31]. It also enhanced GLUT 4 translocation suggesting efficacy in glucose lowering and $\beta$-cell preservation.

Chrysin which is commonly found in honey, pollen, fruits and other medicinal herbs was demonstrated to have reduced the risk of secondary complications of diabetes like neuropathy and nephropathy in high fat diet/Streptozotocin induced diabetic rats besides improving insulin secretion and reduced glucose levels and lipid peroxidation [32].

Baicalein isolated from the roots of Scutellaria baicalensis Georgi and fruits of Oroxylum indicum (L.) Benth was found to significantly improve the hyperglycemia, insulin levels and glucose tolerance in addition to lowering HbA1c levels [33].

30 days administration of Tangeretin, polyphenol abundant in citrus fruit rinds significantly reduced plasma glucose and HbA1c and potentially increased insulin secretion from remnants of $\beta$-cells of pancreas and antioxidants in diabetic rats [34]

Flavonolignans: Silybin, a constituent of Milk thistle (Silybum marianum (L.) Gaertn) has been demonstrated to have beneficial effects against diabetic complications like diabetic neuropathy, nephropathy due to its anti-oxidant nature. It was also supposed to have PPAR $\gamma$ agonist effects [35].

The phenolic nature of flavonolignans is responsible for the antioxidant properties and also the hepato protective activity of silymarin. It also activates regeneration of liver cells and stabilization of cell membrane preventing entry of toxic agents into hepatocytes [36].

\section{Discussion}

Antidiabetic herbs and their compounds were proven to significantly manage glucose intolerance and the associated complications of diabetes mellitus. Flavonoids form the biggest family of polyphenolic herbal compounds which are being demonstrated to possess anti-hyperglycaemic [37], antihyperlipidemic [38], anti-cancerous [39] etc. through their various modes of action both in vitro and in vivo. Flavonoids are of high nutritional value as they form part of our routine diet and hence even high dietary intakes cannot have adverse effects. This could be explained by their rapid metabolism and elimination and also relatively low bioavailability. The biological effects of flavonoids are mainly due to their antioxidant activity and however, the flavonoid metabolites can be able to interact with the cell signalling proteins even after the diminish of antioxidant activity [40]. Complex composition, toxicity risks, targeted delivery to site of action etc. are major obstacles in herbal drugs for clinical investigations. The main drawback with flavonoids in the treatment step is their poor solubility and bioavailability and hence a suitable delivery system is needed to overcome the limitations.

\section{Conclusion and Future Prospects}

Herbal medicine has been targeted to the improvement of human health due to its therapeutic and phytochemical importance but broader application is restricted because of low bioavailability. Still lot of research is needed to explore the herbal medicines, study their safety issues and manifesto clinical trials hoping for a future with "No allopathy-no adversity".

\section{References}

1. Devi VK, Jain N, Valli KS (2010) Importance of novel drug delivery systems in herbal medicines. Pharmacogn Rev 4(7): 27-31.

2. Kumar S, Mishra A, Pandey AK (2013) Antioxidant mediated protective effect of Parthenium hysterophorus against oxidative damage using in vitro models. BMC Complement Altern Med 13: 120.

3. Hanhineva K, Torronen R, Bondia-Pons I, Pekkinen J, Kolehmainen M, et al. (2010) Impact of dietary poly-phenols on carbohydrate metabolism. Int J Mol Sci 11(4): 1365-1402.

4. Katarzyna Małgorzata Brodowska (2017) Natural flavonoids: classification, potential role and application of flavonoid analogues. European Journal of Biological Research 7 (2): 108-123. 
5. Mohan S, Nandhakumar L (2013) Role of various flavonoids: Hypotheses on novel approach to treat diabetes. Journal of Medical Hypotheses and Ideas.

6. Ceriello A (2000) Oxidative stress and glycemic regulation. Metabolism 49 (2 Suppl 1): 27-29.

7. Murni NS, Qamar UA, Alhassan MA, Suganya M, Vikneswari P, et al (2017) Antioxidant and antidiabetic effects of flavonoids: A structure-activity relationship based study. BioMed Research International 2017(2017): $1-14$.

8. Hajiaghaalipour F, Khalilpourfarshbafi M, Arya A (2015) Modulation of glucose transporter protein by dietary flavonoids in type 2 diabetes mellitus. Int J Biol Sci 11: 508-524.

9. Vinson JA, Su X, Zubik L, Bose P (2001) Phenol antioxidant quantity and quality in foods: fruits. J Agric Food Chem 49(11): 5315-5321.

10. Prasath GS, Sundaram CS, Subramanian SP (2013) Fisetin averts oxidative stress in pancreatic tissues of streptozotocin-induced diabetic rats. Endocrine 44(2): 359-568.

11. Bagchi D, Sen CK, Bagchi M, Atalay M (2004) Antiangiogenic, antioxidant, and anti-carcinogenic properties of a novel anthocyanin-rich berry extract formula. Biochemistry (Mosc) 69(1): 75-80.

12. Guo H, Xia M, Zou T, Ling W, Zhong R, et al. (2012) Cyanidin 3-glucoside attenuates obesity-associated insulin resistance and hepatic steatosis in high-fat diet-fed and $\mathrm{db} / \mathrm{db}$ mice via the transcription factor Fox01. Nutr Biochem 23(4): 349-360.

13. Mirshekar M, Roghani M, Khalili M, Baluchnejadmojarad T, Arab MS (2010) Chronic oral pelargonidin alleviates streptozotocin-induced diabetic neuropathic hyperalgesia in rat: Involvement of oxidative stress. Iran Biomed J 14: 33-39.

14. Pari L, Srinivasan S (2010) Antihyperglycemic effect of diosmin on hepatic key enzymes of carbohydrate metabolism in Streptozotocin nicotinamide induced diabetic rats. Biomed Pharmacother. 64: 477-481.

15. Hao HH, Shao ZM, Tang DQ, Lu Q, Chen X, et al. (2012) Preventive effects of Rutin on the development of experimental diabetic nephropathy in rats. Life sci. 91(19-20): 959-967.

16. Prince PSM, Kamalakkannan N (2006) Protective effect of rutin on lipids, lipoproteins, lipid metabolizing enzymes and glycoproteins in streptozotocin-induced diabetic rats. J Pharm Pharmacol 58(10): 13731383.

17. Visnagri A, Kandhare AD, Chakravarty S, Ghosh P, Bodhankar SL (2014) Hesperidin, a flavanoglycone attenuates experimental diabetic neuropathy via modulation of cellular and biochemical marker to improve nerve functions. Pharm Biol 52(7): 814-828.

18. Priscilla DH, Jayakumar M, Thirumurugan K (2015) Flavanone naringenin: An effective anti-hyperglycemic and antihyperlipidemic nutraceutical agent on high fat diet fed streptozotocin induced type 2 diabetic rats. J Funct Foods 14:363-373.

19. Erlund I (2004) Review of the flavonoids quercetin, hesperetin and naringenin. Dietary sources, bioactivities, bioavailability, and epidemiology. Nutr Res 24(10): 851-874.

20. Panchal SK, Poudyal H, Brown L (2012) Quercetin ameliorates cardiovascular, hepatic, and metabolic changes in diet-induced metabolic syndrome in rats. J Nutr 142(6): 1026-1032.

21. Zamora-Ros R, Forouhi NG, Sharp SJ, González CA, Buijsse B, et al. (2014) Dietary intakes of individual flavanols and flavonols are inversely associated with incident type 2 diabetes in European populations. J Nutr 144(3): 335-343.

22. Curtis PJ, Sampson M, Potter J, Dhatariya K, Kroon PA, Cassidy A (2012) Chronic ingestion of flavan-3-ols and isoflavones improves insulin sensitivity and lipoprotein status and attenuates estimated 10-year CVD risk in medicated postmenopausal women with type 2 diabetes: a 1-year, double-blind, randomized, controlled trial. Diabetes Care 35(2): 226-232.

23. Li Y, Ding Y (2012) Minireview: Therapeutic potential of myricetin in diabetes mellitus. Food Sci Human Wellness 1(1): 19-25.

24. Al-Numair KS, Chandramohan G, Veeramani C, Alsaif MA (2015) Ameliorative effect of kaempferol, a flavonoid, on oxidative stress in streptozotocin-induced diabetic rats. Redox Rep 20(5): 198-209.

25. Zang Y, Zhang L, Igarashi $\mathrm{K}, \mathrm{Yu} \mathrm{C}$ (2015) The anti-obesity and antidiabetic effects of kaempferol glycosides from unripe soybean leaves in high-fat-diet mice. Food Funct 6(3): 834-841.

26. Jing Li, Fayong Gong, Fenglin Li (2016) Hypoglycemic and hypolipidemic effects of flavonoids from tatary in type 2 diabetic rats. Biomedical Research 27(1): 132-137.

27. Wang X, Zhang DM, Gu TT, Ding XQ, Fan CY, et al. (2013) Morin reduces hepatic inflammation-associated lipid accumulation in high fructosefed rats via inhibiting sphingosine kinase 1/sphingosine 1-phosphate signalling pathway. Biochem Pharmacol 86(12): 1791-1804.

28. Lee YS, Lee S, Lee HS, Kim BK, Ohuchi K, et al. (2005) Inhibitory effects of isorhamnetin-3-0-beta-D-glucoside from Salicornia herbacea on rat lens aldose reductase and sorbitol accumulation in streptozotocininduced diabetic rat tissues. Biol Pharm Bull 28(5): 916-918.

29. Xinzhou Yang, Jing Yang, Chan Xu, Mi Huang, Qi Zhou, et al. (2015) Antidiabetic effects of flavonoids from Sophora flavescens EtOAc extract in type 2 diabetic KK-ay mice. J Ethnopharmacol 171: 161-170.

30. Fu Z, Gilbert ER, Pfeiffer L, Zhang Y, Fu Y, et al. (2012) Genistein ameliorates hyperglycemia in a mouse model of nongenetic type 2 diabetes. Appl Physiol Nutr Metab 37(3): 480-488.

31. Panda S, Kar A (2007) Apigenin (4,5,7-trihydroxyflavone) regulates hyperglycaemia, thyroid dysfunction and lipid peroxidation in alloxaninduced diabetic mice. J Pharm Pharmacol 59(11): 1543-1548.

32. Fraschini F, Demartini G, Esposti D (2002) Pharmacology of silymarin Clin Drug Invest 22(1): 51-65.

33. Sirovina D, Orsolić N, Koncić MZ, Kovacević G, Benković V, et al. (2013) Quercetin vs chrysin: Effect on liver histopathology in diabetic mice. Hum Exp Toxicol 32(10): 1058-1066.

34. Stavniichuk R, Drel VR, Shevalye H, Maksimchyk Y, Kuchmerovska TM, et al. (2011) Baicalein alleviates diabetic peripheral neuropathy through inhibition of oxidative-nitrosative stress and p38 MAPK activation. Exp Neurol 230(1): 106-113.

35. Sundaram R, Shanthi P, Sachdanandam P (2014) Effect of tangeretin, a polymethoxylated flavone on glucose metabolism in streptozotocininduced diabetic rats. Phytomedicine 21(6): 793-799.

36. Kazazis CE, Evangelopoulos AA, Kollas A, Vallianou NG (2014) The Therapeutic Potential of Milk Thistle in Diabetes. Rev Diabet Stud 11(2): 167-174.

37. Grassi D, Desideri G, Necozione S, Lippi C, Casale R, Properzi G (2008) Blood pressure is reduced and insulin sensitivity increased in glucoseintolerant, hypertensive subjects after 15 days of consuming highpolyphenol dark chocolate. J Nutr 138(9): 1671-1676.

38. Flammer AJ, Sudano I, Wolfrum M, Thomas R, Enseleit F, Périat D (2012) Cardiovascular effects of flavanol-rich chocolate in patients with heart failure. Eur Heart J 33(17): 2172-2180.

39. Yang CS, Yang GY, Landau JM, Kim S, Liao J (1998) Tea and tea polyphenols inhibit cell hyper-proliferation, lung tumorigenesis, and tumor progression. Exp Lung Res 24(4): 629-639.

40. Spencer JP, Rice-Evans C, Williams RJ (2003) Modulation of pro-survival Akt/protein kinase B and ERK1/2 signaling cascades by quercetin and its in vivo metabolites underlie their action on neuronal viability. J Biol Chem 278(37): 34783-34793. 